\title{
High working memory capacity attenuates the deviation effect but not the changing-state effect: Further support for the duplex-mechanism account of auditory distraction
}

\author{
PATRIK SörQvist \\ University of Gävle, Gävle, Sweden
}

\begin{abstract}
Serial short-term memory is impaired by background sound, at least when a sound element suddenly deviates from an otherwise repetitive sequence (the deviation effect) and when each sound element in the sequence differs from the preceding one (the changing-state effect). Two competing theories have been proposed to explain these effects: One suggests that both effects are caused by the same mechanism (i.e., attentional resources being depleted by the sound), and the other suggests that the deviation effect is caused by attentional capture and that the changingstate effect is caused by interference between order processes. The present investigation found that working memory capacity predicts susceptibility to the deviation effect, but not to the changing-state effect, both when speech items (Experiment 1) and when tones (Experiment 2) produce the disruption. These results suggest that the two effects are caused by different mechanisms and support the duplex-mechanism account of auditory distraction.
\end{abstract}

As is well known, serial short-term memory of visually presented items is impaired by the mere presence of background sounds (Colle \& Welsh, 1976; Ellermeier \& Zimmer, 1997; Jones \& Macken, 1993; Macken, Tremblay, Alford, \& Jones, 1999; Salamé \& Baddeley, 1982), at least when the sound sequence changes acoustically. For instance, the $[\mathrm{k} 1 \mathrm{~m} \mathrm{v} \mathrm{r} \mathrm{q} \mathrm{c]} \mathrm{sound} \mathrm{sequence} \mathrm{is} \mathrm{more}$ distracting to serial recall than is the [c $\mathrm{c} \mathrm{c} \mathrm{c} \mathrm{c} \mathrm{c} \mathrm{c]} \mathrm{sound}$ sequence. This finding is called the changing-state effect (Jones \& Macken, 1993; Macken et al., 1999). Furthermore, an auditory event that stands out or deviates from the recent auditory past, such as the sound $/ \mathrm{m} /$ in the [c c c m c c c] sound sequence, disrupts serial recall. This phenomenon is known as the deviation effect (Hughes, Vachon, \& Jones, 2005, 2007; Lange, 2005; see also Parmentier, 2008).

The interference-by-process account has been proposed as a possible explanation of the changing-state effect (Macken et al., 1999). According to this theory, the changing-state effect is caused by interference between two sets of order processes: (1) the automatic processing of order between successive and perceptually discrete sound events and (2) the deliberate processing of the order between the to-be-serially-recalled items. On the basis of this theory, then, the changing-state effect takes place only when the primary task requires order processing (e.g., serial rehearsal of visual verbal items).

Cowan (1995) has proposed another possibility. According to his theory, changes in a sound stream deplete attentional resources that could otherwise be used to entertain the focal task. In other words, each new sound element in a changing-state sound stream (e.g., $\mathrm{k} 1 \mathrm{~m} \mathrm{v} \mathrm{r} \mathrm{q} \mathrm{c)}$ attracts orienting responses (Öhman, 1979; Siddle, 1991; Sokolov, 1963) accompanied by a redirection of attention toward the new stimulus (away from the focal task) and causes disruption. The same mechanism could also explain the deviation effect: The $/ \mathrm{m} /$ sound element in the [c c c m c c c] sound sequence elicits an orienting response and disrupts performance as a consequence.

Due to its parsimonious character, Cowan's (1995) theory is attractive, and, at first glance, it may seem like a changing-state sound sequence is just a series of deviant sounds, in that each item deviates from the preceding item. However, a number of findings suggest that changing-state and deviating sounds have different effects on cognitive performance. First, a deviating sound disrupts performance on tasks that do not require any order processing (see, e.g., Berti \& Schröger, 2003; Hughes et al., 2007; Parmentier, 2008), but changing-state sound sequences do not (Hughes et al., 2007; Jones \& Macken, 1993; Perham, Banbury, \& Jones, 2007). Second, the changing-state effect and the deviation effect do not interact in their disruption of serial recall (Hughes et al., 2007). Third, the deviation effect is absent when the deviant is presented during a retention interval between encoding and retrieval, whereas the changing-state effect is still present when the sound is presented during the retention interval (Hughes et al., 2005). Fourth, and finally, a large body of evidence has 
demonstrated habituation toward the disruptive effects of deviating sounds on task performance (e.g., Debener, Kranczioch, Herrmann, \& Engel, 2002; Friedman, Cycowicz, \& Gaeta, 2001; Siddle, 1991; Sokolov, 1963), but people seem unable to habituate to the effects of changingstate sound sequences on serial recall (Ellermeier \& Zimmer, 1997; Jones, Macken, \& Mosdell, 1997; Tremblay \& Jones, 1998). These results lean toward the possibility that the changing-state effect and the deviation effect are caused by qualitatively different mechanisms. Because of this, Hughes et al. (2007) proposed a duplex-mechanism account of auditory distraction. According to this view, the changing-state effect is the result of interference between order processes, whereas the deviation effect is caused by attentional capture. The purpose of the present study was to investigate the nature of the mechanisms that underlie individual differences in susceptibility to these two effects.

Complex span tasks are typically employed to investigate individual differences in cognitive capacity. In a classic complex span task called operation span (OSPAN), the present participants were presented with sets of operation word strings [e.g., "Is $(4 \times 3)+5=17$ ? CACTUS"] and were requested to respond yes or no to the operation and to remember the word for later recall. After responding to a set of those operation word strings, the participants recalled the words. The score on the recall part of the task was used to measure what is called working memory capacity (WMC). A large number of studies have shown that WMC is extremely successful in predicting performance across a wide range of domains (for reviews, see Engle, 2002; Unsworth \& Engle, 2007), including the antisaccade task (Kane, Bleckley, Conway, \& Engle, 2001; Unsworth, Schrock, \& Engle, 2004), Eriksen's flanker task (Heitz \& Engle, 2007), the attention network test (Redick \& Engle, 2006), and the Stroop task (Kane \& Engle, 2003). Findings such as these have led some authors to argue that WMC measures individual differences in a general and limited pool of attentional resources that can be used to inhibit task-irrelevant information and constrain attention to the primary task (Engle, 2002; Kane et al., 2001; Lustig, Hasher, \& Zacks, 2007).

If the changing-state effect is caused by depletion of attentional resources, as was suggested by Cowan (1995), there should be a relationship between WMC and the magnitude of the changing-state effect. However, several authors have tried and failed to find this relationship (Beaman, 2004; Elliott \& Cowan, 2005; for an exception, see Elliott, Barrilleaux, \& Cowan, 2006). On the other hand, if the changing-state effect is caused by interference between order processes, as is proposed by the interferenceby-process account (Macken et al., 1999), then individual differences in the capability to process order, rather than WMC, should predict disruption. To support this claim, Macken, Phelps, and Jones (2009) presented participants with pairs of sound patterns and asked them to judge whether or not the two patterns in each pair were the same. The authors argued that the pattern-matching task measures the capability to automatically process order information in sound sequences, and they were able to show that the participants who performed well on the pattern- matching task were most susceptible to the changing-state effect. The authors concluded that the magnitude of the changing-state effect is a function of the efficiency by which people process the order between individual sound elements, not a function of an all-purpose pool of attentional resources (i.e., WMC).

In contrast to those studies, a handful of experiments have shown that WMC does indeed predict susceptibility to some types of auditory distraction that are not evidently dependent on a conflict between order processes. For instance, Beaman (2004) asked participants to view lists of visual to-be-remembered words (e.g., tools) and recall the words in free order; thereafter, he presented lists of to-be-ignored speech words that were semantically related to the to-be-remembered words (e.g., other tools). At recall, low-WMC individuals reported more of the to-be-ignored speech words than high-WMC individuals did. Other studies have shown that people with high WMC are less susceptible to the detrimental effects of speech and aircraft noise on reading comprehension and prose memory (Sörqvist, 2010; Sörqvist, Halin, \& Hygge, 2010; Sörqvist, Ljungberg, \& Ljung, 2010).

Besides these demonstrations of relationships between WMC and auditory distraction, a study by Conway, Cowan, and Bunting (2001) is of particular interest to the present investigation. When engaged in a conversation at a party, hearing your own name spoken in the background can capture your attention (i.e., the cocktail party phenomenon). Conway et al. (2001) investigated the role of WMC in this phenomenon by requesting that participants continuously repeat aloud (i.e., shadow) a message presented to one ear while ignoring another message presented to the other ear. The participant's own name was spoken in the to-be-ignored message at some point during shadowing. The results revealed that high-WMC individuals were less likely to report hearing their own name. Furthermore, they were less likely to make shadowing mistakes when their name was presented. Since the deviation effect seems to be a result of attentional capture (e.g., Hughes et al., 2007), similar to the cocktail party phenomenon, Conway et al.'s (2001) results suggest that WMC should predict the magnitude of the deviation effect.

The possibilities that susceptibility to the changingstate effect is a function of order processes (Macken et al., 2009) and that susceptibility to the cocktail party phenomenon is a function of WMC (Conway et al., 2001) fit nicely with the duplex-mechanism account of auditory distraction (Hughes et al., 2007). To recollect, this account proposes that the deviation effect is caused by attentional capture, and if WMC reflects some general pool of attentional resources (see, e.g., Kane et al., 2001), then high WMC should attenuate the deviation effect. In contrast, WMC should not predict the magnitude of the changingstate effect, because this effect is caused by interference between order processes. The present investigation tested these hypotheses in two experiments. In Experiment 1, the disruption of serial recall was produced by speech sounds. In Experiment 2, the disruption was produced by tones in order to determine whether the same pattern of results would generalize across speech and nonspeech sounds. 


\section{EXPERIMENT 1}

\section{Method}

\section{Participants}

A total of 40 university students (mean age $=23.35$ years, $S D=$ 5.27) participated in Experiment 1 in exchange for a small honorarium. They all reported having Swedish as their native language, normal hearing, and normal or corrected-to-normal vision.

\section{Apparatus and Materials}

OSPAN. A computerized version of the OSPAN task developed by Turner and Engle (1989) was adopted. In this task, mathematical operations - such as "Is $(6+4) \times 2=20$ ?" - were presented on a computer screen. The participants were asked to answer yes or $n o$ to the question by pressing a button on the keyboard. They were encouraged to respond as accurately and quickly as possible. After the buttonpress, the screen went blank. A one-syllable noun (e.g., CAT), which the participants were told to remember for later recall, was then presented on the screen for $1 \mathrm{sec}$. When the to-beremembered word disappeared, either a new mathematical operation was presented or the list ended, depending on the length of the list. The list length (i.e., the number of words to be remembered) varied between two and six words. The words within a list were semantically unrelated, and each word was presented only once during the task. When the last word in each list had been presented, the participants were instructed to recall as many of the words as they could remember, in the order of presentation, by typing on the computer keyboard. The total number of lists was 16 (i.e., 3 of each list length and 1 for practice), and the lists were presented in the same random order for each participant. The task was scored using a proportion procedure (see Conway et al., 2005), in which credit was given for each word recalled in the correct serial position. The total number of words accurately recalled in each list was then divided by the list length. For instance, if the participants recalled all four words in the correct serial position on a four-word list, they would receive one point for that list; but if four out of five words were recalled on a five-word list, they would receive 0.80 points for that list. These proportions were thereafter averaged over the total number of lists (i.e., 15).

Serial recall. The to-be-remembered, visually presented sequences consisted of eight digits taken without replacement from the set 1-9. They were arranged in a pseudorandom order (successive digits were not arithmetically adjacent). Each digit was presented for $350 \mathrm{msec}$ with an interstimulus interval of $400 \mathrm{msec}$ (similar to in Hughes et al., 2007).

A set of 4 spoken letters (i.e., c, k, m, j) was recorded in an evenpitched male voice with Swedish pronunciation and was sampled at a 32-bit resolution at a sampling rate of $44100 \mathrm{~Hz}$. The spoken items were digitally edited to last $200 \mathrm{msec}$ in length. Three types of task-irrelevant sound sequences were composed from this set of spoken letters using computer software. In a steady-state sequence, the spoken letter "c" was presented 21 times. Each item was separated by a $100-\mathrm{msec}$ silent pause. A deviant sequence was identical to a steady-state sequence with the exception that the 11th item was the spoken letter " $k$ " instead of "c." A changing-state sequence was identical to a steady-state sequence, except that it consisted of 21 spoken letters taken from the set of 4 and repeated systematically (e.g., c, k, m, j, c, k, m, j, c . . c). The onset of the first spoken letter, identical for all three types of sound sequences, occurred $300 \mathrm{msec}$ before the first to-be-remembered digit. The 11th spoken letter was presented between the fourth and the fifth to-be-remembered digits, and the final spoken letter was presented $100 \mathrm{msec}$ after the eighth to-be-remembered digit.

The participants were asked to recall the digits in order of presentation $500 \mathrm{msec}$ after the final spoken letter had been presented. The participants were instructed to always recall eight digits, to be sure to place the digits in the correct serial position, and, if they did not know which digit should be where in the sequence, to guess. Recall was self-paced and made by typing with the computer keyboard.
When the participants had recalled the digits, they pressed a button that initiated the next trial.

\section{Design and Procedure}

A within-participants design was used. The participants were tested individually in a sound-attenuated room. The presentation of test materials was made by a computer, and the sound (at a 32-bit resolution and $44100-\mathrm{Hz}$ sampling rate and at approximately $65 \mathrm{~dB}[\mathrm{~A}]$, as measured by a Brüel \& Kjaer sound-level meter) was played through headphones (Sennheiser HD 202) attached to the computer, which used a Soundmax Digital HD Audio soundcard. The participants were instructed to ignore any sound they would hear in the headphones. All participants began with performing the OSPAN task. Thereafter, they performed two blocks of 30 serial recall trials. In one block (the deviant block), the first 2 trials were steady-state trials used to familiarize the participants with the task. Performance on these trials was excluded from the analysis. The remaining 28 trials consisted of 22 steady-state trials and 6 deviant trials. The deviant trials occurred at Trials 5, 9, 15, 21, 24, and 29. The other block (the changing-state block) was identical to the deviant block, with the exception that changing-state sound sequences were played instead of deviant sound sequences at Trials 5, 9, 15, 21, 24 , and 29. The two blocks were separated by a self-paced pause, and the order of the two blocks was counterbalanced across participants: Half began with the deviant block, and the other half began with the changing-state block. The experimental session lasted approximately $30 \mathrm{~min}$.

\section{Results}

\section{OSPAN}

The participants' mean score on the OSPAN task (in proportion) was .76 (range $=.41-.97, S D=.14)$. The mean score on the operation part of the task (in proportion) was .92 (range $=.77-1.00, S D=.06$ ). A correlation analysis revealed a positive and significant correlation between OSPAN score and operation performance $[r(38)=$ $.32, p<.05]$. This is fortunate because it indicates that the participants who scored high on the memory part of the task did not do so as a result of having skipped the operation part of the task.

\section{Serial Recall}

The responses on the serial recall task were scored according to a strict serial recall criterion (points were given only to correct items placed in the correct serial position). Since there were more steady-state trials than other types of trials, the score was calculated as the percentage of digits correctly recalled within each sound condition and type of block (deviant or changing-state), respectively.

The deviant block. In this section, the data were taken only from the deviant block. As can be seen in Figure 1, the deviant reduced recall across most serial positions. A 2 (background condition: steady-state vs. deviant) $x$ 8 (serial position) ANOVA revealed significant main effects of background condition $\left[F(1,39)=47.26, M S_{\mathrm{e}}=\right.$ $\left.0.04, p<.001, \eta^{2}=.55\right]$ and serial position $[F(7,273)=$ $\left.34.01, M S_{\mathrm{e}}=0.05, p<.001, \eta^{2}=.47\right]$ and a significant interaction between the two variables $[F(7,273)=4.08$, $\left.M S_{\mathrm{e}}=0.02, p<.01, \eta^{2}=.10\right]$.

The common way to measure individual differences in susceptibility to auditory distraction is to take the difference between the scores in the control and distraction conditions (Ellermeier \& Zimmer, 1997; Macken et al., 2009). 


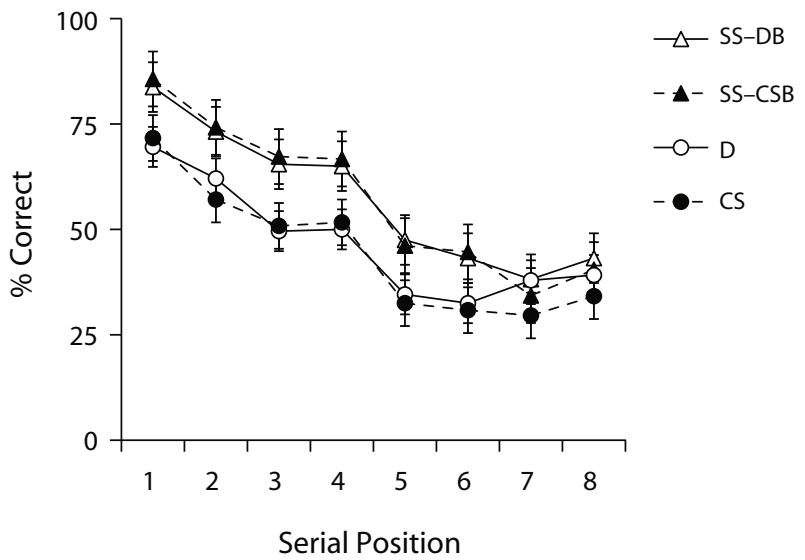

Figure 1. Mean percentages of items correctly recalled in each serial position in the steady-state condition in the deviant block (SS-DB), the steady-state condition in the changing-state block (SS-CSB), the deviant condition (D), and the changing-state condition (CS) in Experiment 1. Error bars show standard errors of the means.

This method was used here as well. The mean difference score was $0.11(S D=0.10)$. The correlation between OSPAN scores and the difference scores was significant and negative $[r(38)=-.35, p<.05]$, which indicates that high WMC attenuates the deviation effect. This relationship is illustrated in Figure 2. There is reason to believe that using difference scores is a less sophisticated statistical analysis than using residual analysis is, because the error measurement of difference scores is larger than the error of residuals (Cronbach \& Furby, 1970). Therefore, a hierarchal regression analysis with scores in the deviant condition as the dependent variable, scores in the steadystate condition as the independent variable in the first step, and OSPAN scores as the independent variable in the second step was calculated. The analysis revealed significant regression models in the first $\left[R^{2}=.70 ; F(1,38)=89.65\right.$, $\left.M S_{\mathrm{e}}=0.01, p<.001\right]$ and second $\left[R^{2}=.77 ; F(2,37)=\right.$ $\left.60.48, M S_{\mathrm{e}}=0.01, p<.001\right]$ steps. The scores in the steady-state condition explained a significant part of the variance $(\beta=.73 ; t=8.49, p<.001)$ in the second step of the analysis, and the OSPAN score explained a significant part of the residual variance not explained by the scores in the steady-state condition $(\beta=.27 ; t=3.17$, $p<.005)$. The residual analysis is, therefore, consistent with the analysis that was based on difference scores: High WMC attenuates the deviation effect.

The changing-state block. In this section, the data were taken from the changing-state block only. As can be seen in Figure 1, the changing-state effect was replicated. A 2 (background condition: steady-state vs. changingstate) $\times 8$ (serial position) ANOVA revealed significant main effects of background condition $[F(1,39)=57.61$, $\left.M S_{\mathrm{e}}=0.04, p<.001, \eta^{2}=.60\right]$ and serial position $\left[F(7,273)=48.44, M S_{\mathrm{e}}=0.05, p<.001, \eta^{2}=.55\right]$ and a significant interaction between the variables $[F(7,273)=$ 2.57, $\left.M S_{\mathrm{e}}=0.02, p<.05, \eta^{2}=.06\right]$.

To obtain a measure of individual differences in susceptibility to the changing-state effect, the difference between the scores in the steady-state condition and the scores in the changing-state condition was calculated. The mean difference score was $0.13(S D=0.11)$. The correlation between OSPAN scores and the difference scores was negative but nonsignificant $[r(38)=-.14, p=.40]$. Hence, there is no evidence of a relationship between WMC and the changing-state effect. Following the advice of Cronbach and Furby (1970), I calculated a hierarchal regression analysis with scores in the changing-state condition as the dependent variable, scores in the steady-state condition as the independent variable in the first step, and OSPAN score as the independent variable in the second step. The analysis revealed a significant regression model in the first $\left[R^{2}=.64 ; F(1,38)=68.67, M S_{\mathrm{e}}=0.01, p<\right.$ $.001]$ and second $\left[R^{2}=.67 ; F(2,37)=37.13, M S_{\mathrm{e}}=0.01\right.$, $p<.001]$ steps. The scores in the steady-state condition explained a significant part of the variance $(\beta=.74 ; t=$ $7.13, p<.001)$ in the second step, but the OSPAN score did not explain a significant part of the residual variance ( $\beta=.17 ; t=1.62, p=.11)$. The regression analysis is, therefore, consistent with the analysis using difference scores: No evidence of a relationship between WMC and the changing-state effect was revealed.

\section{Discussion}

The results of Experiment 1 indicate that high WMC attenuates the deviation effect but not the changing-state effect. Even though the difference-score analysis clearly proposes that WMC is unrelated to the changing-state effect, the residual analysis points to the possibility that WMC could be related to the changing-state effect, provided enough statistical power, even though this relationship should be much weaker than the relationship between WMC and the deviation effect. Furthermore, previous studies indicate that WMC might be related to the changing-state effect when produced by tone sequences rather than by spokenletter sequences (e.g., Elliott et al., 2006). Because of this, in Experiment 2, I intended to determine whether the pattern of results found in Experiment 1 replicates when tone sequences produce the disruption.

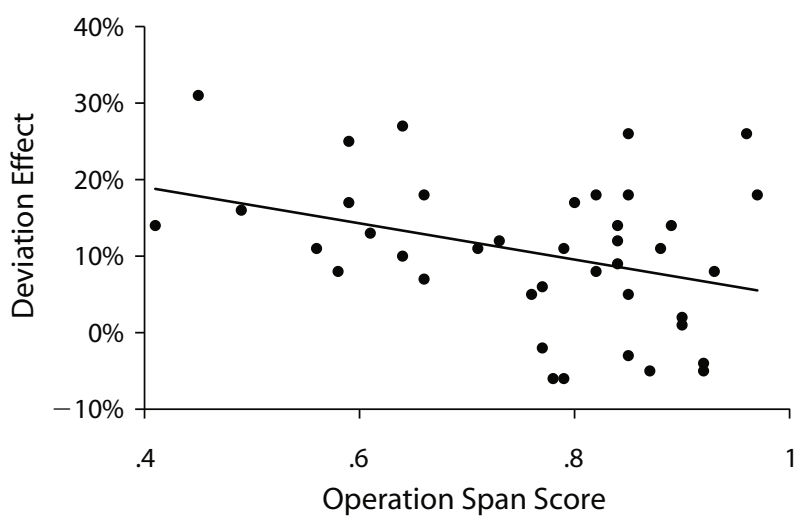

Figure 2. The correlation between operation span score and the deviation effect (calculated as the difference between serial recall score in the steady-state condition and serial recall score in the deviant condition) in Experiment 1. 


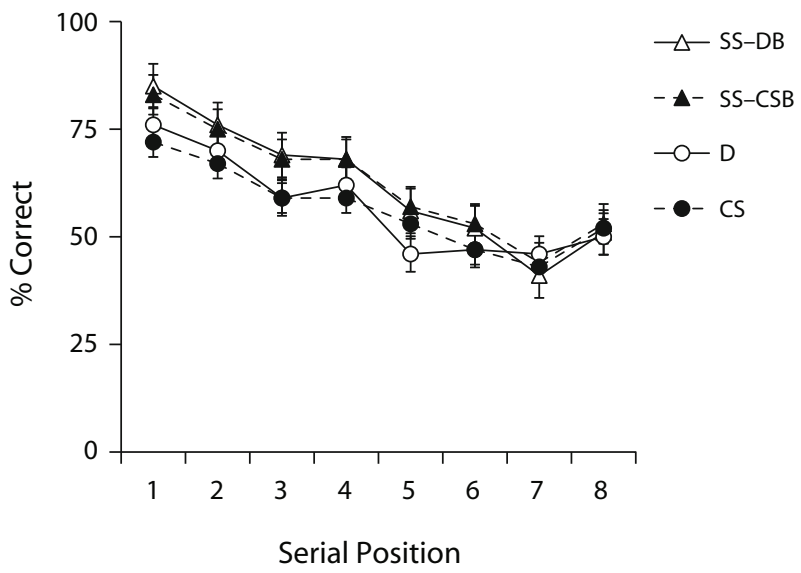

Figure 3. Mean percentages of items correctly recalled in each serial position in the steady-state condition in the deviant block (SS-DB), the steady-state condition in the changing-state block (SS-CSB), the deviant condition (D), and the changing-state condition (CS) in Experiment 2. Error bars show standard errors of the means.

\section{EXPERIMENT 2}

\section{Method}

\section{Participants}

A total of 48 university students (mean age $=24.46$ years, $S D=$ 3.90) participated in Experiment 2 in exchange for a small honorarium. They all reported having Swedish as their native language, normal hearing, and normal or corrected-to-normal vision. None of the participants in Experiment 2 had participated in Experiment 1.

\section{Apparatus and Materials}

The materials were identical to those in Experiment 1, except for the sound sequences. In Experiment 2, three types of tone sequences were generated with a computer. In the steady-state sequence, 21 tones were presented. The tones were played at $440 \mathrm{~Hz}$, were $200 \mathrm{msec}$ in length (with $10-\mathrm{msec}$ rise and fall times), and were separated by a $100-\mathrm{msec}$ silent pause. The deviant sequence was identical to the steady-state sequence, with the exception that the 11 th tone was played at $220 \mathrm{~Hz}$ instead of at $440 \mathrm{~Hz}$. The changingstate sequence was identical to the steady-state sequence, except that it consisted of 21 tones taken from a set of four frequencies $(220$, $440,880$, and $1760 \mathrm{~Hz})$ and repeated systematically $(440,880,220$, 1760, 440, $880 \mathrm{~Hz}$, etc.).

\section{Design and Procedure}

The design, procedure, and when and how the sound sequences were presented were identical to those in Experiment 1.

\section{Results}

\section{OSPAN}

The participants' mean score on the OSPAN task (in proportion) was .79 (range $=.40-.99, S D=.15)$. The mean score on the operation part of the task (in proportion) was .94 (range $=.80-1.00, S D=.05$ ). A correlation analysis revealed a positive and significant correlation between OSPAN score and operation performance $[r(46)=$ $.28, p=.05]$. Hence, as in Experiment 1, the participants who scored high on the memory part of the task did not do so as a result of having skipped the operation part of the task.

\section{Serial Recall}

The serial recall task was scored the same way as in Experiment 1.

The deviant block. In this section, the data were taken from the deviant block only. As can be seen in Figure 3, the deviation effect was replicated. A 2 (background condition: steady-state vs. deviant) $\times 8$ (serial position) ANOVA revealed significant main effects of background condition $\left[F(1,47)=12.95, M S_{\mathrm{e}}=0.04, p<.001, \eta^{2}=\right.$ $.22]$ and serial position $\left[F(7,329)=25.73, M S_{\mathrm{e}}=0.06\right.$, $\left.p<.0001, \eta^{2}=.35\right]$ and a significant interaction between the two variables $\left[F(7,329)=4.04, M S_{\mathrm{e}}=0.02, p<.001\right.$, $\left.\eta^{2}=.08\right]$.

The mean difference score between the steady-state and deviation conditions was $0.05(S D=0.10)$. The correlation between the OSPAN scores and the difference scores was significant and negative $[r(46)=-.31, p<.05]$, indicating that high WMC attenuated the deviation effect. This relationship is illustrated in Figure 4. The differencescore analysis was complemented by a hierarchal regression analysis, following the statistical advice of Cronbach and Furby (1970). Scores in the deviant condition constituted the dependent variable in this analysis. Scores in the steady-state condition constituted the independent variable in the first step, and OSPAN score was the independent variable in the second step. The regression model was significant in the first $\left[R^{2}=.76 ; F(1,46)=143.75, M S_{\mathrm{e}}=\right.$ $0.01, p<.01]$ and second $\left[R^{2}=.79 ; F(2,45)=84.91\right.$, $\left.M S_{\mathrm{e}}=0.01, p<.01\right]$ steps. The scores in the steady-state condition explained a significant part of the variance $(\beta=$ $.79 ; t=10.32, p<.01)$ in the second step of the analysis, and the OSPAN score explained a significant part of the residual variance not explained by the scores in the steady-state condition $(\beta=.20 ; t=2.57, p<.05)$. The residual analysis is, therefore, consistent with the analysis that was based on difference scores: High-WMC individuals are less susceptible to the deviation effect.

The changing-state block. In this section, the data were taken from the changing-state block only. As can be

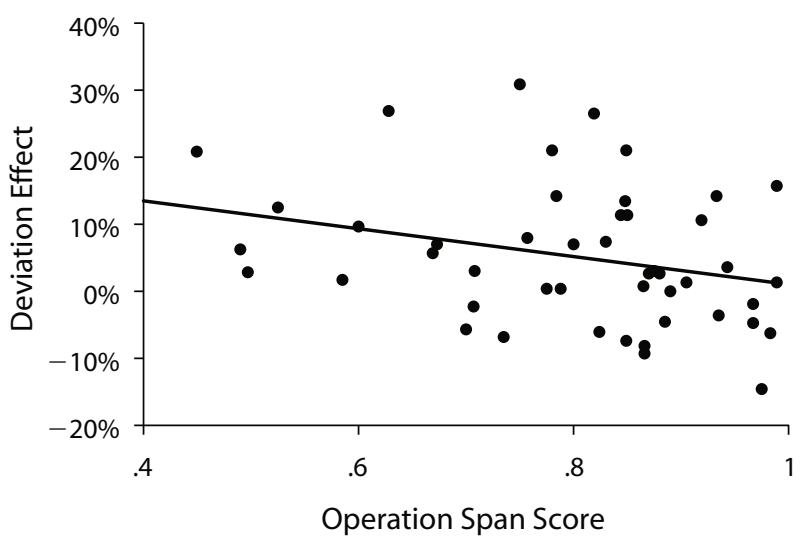

Figure 4. The correlation between operation span score and the deviation effect (calculated as the difference between serial recall score in the steady-state condition and serial recall score in the deviant condition) in Experiment 2. 
seen in Figure 3, the changing-state effect was replicated. A 2 (background condition: steady-state vs. changingstate) $\times 8$ (serial position) ANOVA revealed significant main effects of background condition $[F(1,47)=13.41$, $\left.M S_{\mathrm{e}}=0.05, p<.001, \eta^{2}=.22\right]$ and serial position $\left[F(7,329)=18.01, M S_{\mathrm{e}}=0.07, p<.0001, \eta^{2}=.28\right]$, and the interaction between the variables approached significance $\left[F(7,329)=1.88, M S_{\mathrm{e}}=0.02, p=.07\right.$, $\left.\eta^{2}=.04\right]$.

The mean difference score between the steady-state condition and the changing-state condition was 0.06 $(S D=0.11)$. The correlation between the OSPAN scores and the difference scores was positive but nonsignificant $[r(46)=.19, p=.20]$. Hence, there was no evidence of a relationship between WMC and the changing-state effect. If anything, the correlation analysis indicated that highWMC individuals are slightly, but far from significantly, more susceptible to the changing-state effect. Following the advice of Cronbach and Furby (1970), I calculated a hierarchal regression analysis with scores in the changingstate condition as the dependent variable, scores in the steady-state condition as the independent variable in the first step, and OSPAN scores as the independent variable in the second step. The analysis revealed a significant regression model in the first $\left[R^{2}=.66 ; F(1,46)=90.89\right.$, $\left.M S_{\mathrm{e}}=0.01, p<.01\right]$ and second $\left[R^{2}=.67 ; F(2,45)=\right.$ 46.04, $\left.M S_{\mathrm{e}}=0.01, p<.01\right]$ steps. The scores in the steady-state condition explained a significant part of the variance $(\beta=.87 ; t=8.72, p<.01)$ in the second step, but the OSPAN score did not explain a significant part of the residual variance $(\beta=-.10 ; t=-1.03, p=.31)$.

\section{Discussion}

Experiment 2 replicated the relationship between WMC and the deviation effect found in Experiment 1. Furthermore, WMC was once again unrelated to the changingstate effect. In fact, in Experiment 2, the relationship between WMC and the deviation effect $(r=-.31)$ differed significantly from the relationship between WMC and the changing-state effect $(r=.19)[t(45)=3.02, p<.01]$, which provides relatively strong evidence that the two effects are produced by different mechanisms.

\section{GENERAL DISCUSSION}

The investigation reported here is the first to demonstrate a relationship between WMC and the deviation effect. This finding accords with previous studies that showed that high WMC can attenuate the power of sounds to capture attention (Conway et al., 2001). The experiments reported here also found that WMC is unrelated to the changing-state effect, a finding that is consistent with those from previous research (Beaman, 2004; Elliott \& Cowan, 2005). This pattern of results was the same whether spoken letters (Experiment 1) or tones (Experiment 2) constituted the sound sequences that produced the disruption of the serial recall task.

From the results reported here, it seems safe to conclude that at least two mechanisms underlie individual differ- ences in susceptibility to auditory distraction. One of those mechanisms is measured by WMC and contributes to several auditory distraction phenomena (Beaman, 2004; Conway et al., 2001; Sörqvist, 2009; Sörqvist, Halin, \& Hygge, 2010; Sörqvist, Ljungberg, \& Ljung, 2010), including the deviation effect. The other mechanism is the efficiency by which people process the order between successive and perceptually discrete sound events, which contributes to individual differences in susceptibility to the changingstate effect (Macken et al., 2009). The observation that two different mechanisms underlie the deviation effect and the changing-state effect is especially supportive of the duplex-mechanism account by Hughes et al. (2007), according to which the two effects have qualitatively different origins. Taken together, the results suggest that the changing-state effect is caused by interference between order processes, whereas the deviation effect is caused by some mechanism related to attention switching.

There are, however, alternative interpretations of the results reported here that should be addressed. For instance, it could be argued that high-WMC individuals can control attention switching toward deviating sounds as long as the degree of deviation is relatively small (as with sound sequences that include a single deviant). A changingstate sound sequence, however, involves so much acoustic change that it breaks through high-WMC individuals' ability to control orienting responses toward the sounds. In other words, the deviation effect and the changing-state effect may differ only to the degree by which they capture attention, not in quality. This could explain the absence of a relationship between WMC and the changing-state effect and would be consistent with theories assuming similar mechanisms for both effects (cf. Cowan, 1995). However, there are a number of reasons to doubt this alternative interpretation. Hughes et al. $(2005,2007)$ have shown that deviating sounds and changing-state sounds have qualitatively different effects on cognitive performance. In addition to Hughes et al.'s $(2005,2007)$ findings, the present investigation shows that the magnitude of the two effects is approximately equal (see Figures 1 and 3 ). Were the two effects produced by the same mechanism, the changingstate sound sequences should have caused a substantially larger degree of disruption.

It should be noted that Elliott et al. (2006) found highWMC individuals to be less susceptible to the changingstate effect when it was produced by tone sequences. However, there are reasons to question whether this finding points to a relationship between WMC and the classic changing-state effect. First, the authors failed to find a similar relationship with spoken-letter sequences in the same experiment. This is alarming because a large body of evidence suggests that tone sequences and spoken-letter sequences produce qualitatively equal (changing-state) effects on serial recall (e.g., Jones \& Macken, 1993; Tremblay, Nicholls, Alford, \& Jones, 2000; for a review, see Macken et al., 1999). Second, a handful of experiments have failed to find relationships between memory capacity and the changing-state effect (Beaman, 2004; Ellermeier \& Zimmer, 1997; Neath, Farley, \& Surprenant, 2003), including the present experiments and an experiment (El- 
liott \& Cowan, 2005) using the exact same tone sequences as those used by Elliott et al. Furthermore, if the deviation effect and the changing-state effect are indeed caused by a mechanism related to $\mathrm{WMC}$, it is difficult to see why the investigation reported here indicates that the relationship between WMC and the deviation effect is significantly different from the relationship between WMC and the changing-state effect. Taken together, the weight of evidence supports the argument that WMC is not related to the changing-state effect.

Another contribution from the present investigation is worth highlighting. Previous studies of the deviation effect in short-term memory have used speech sounds to produce the disruption (Hughes et al., 2005, 2007). The present study extended the deviation effect in short-term memory to nonspeech sounds (but see Lange, 2005, for a slightly different way of producing the deviation effect with nonspeech sounds). This result is not surprising, because deviation effects have been produced with nonspeech sounds in other paradigms (e.g., Berti \& Schröger, 2003; Parmentier, 2008). Yet, the results are important because they complicate the phenomenon for theories trying to explain auditory distraction of short-term memory in terms of the structural similarities between the content of the task-relevant and task-irrelevant materials (e.g., Neath, 2000; Salamé \& Baddeley, 1982). Since verbal memory materials and tones have fairly little content in common, those theories cannot adequately explain why deviating tones disrupt serial verbal short-term memory.

Finally, I turn to the implications of the results presented here to the nature of WMC. In a recent review of the WMC literature, Unsworth and Engle (2007) concluded that individual differences in WMC reflect differences in two mechanisms: (1) the ability to retrieve items from secondary memory, and (2) the ability to actively maintain items in primary memory in the presence of interference. This conceptualization of WMC is consistent with the older views of WMC as being essentially a measure of domaingeneral attentional control and resistance to interference (Cowan, 2005; Engle, 2002; Kane et al., 2001; Lustig et al., 2007). The relationship between WMC and the deviation effect can be explained by high-WMC individuals' being more efficient in retrieving the target items from secondary memory when attention has been diverted by the deviant. However, the proposition that WMC also reflects a general ability to resist interference is complicated by several problems from the results reported here. First, parts of the relationship between WMC and the deviation effect seem to have arisen due to high-WMC individuals' performing slightly better in the presence of the deviant (see Figures 2 and 4). Hence, high-WMC individuals seem not only better able to resist interference, but also more efficient at inhibiting the act of diverting attention when a deviant is presented, and this inhibition process could accompany increased activation of target items. Another possibility is that a deviant increases high-WMC individuals' arousal, and their ability to focus on the target items increases as a consequence, a view that would be consistent with some other studies of positive effects of noise on cognitive performance (Sikström \& Söderlund, 2007).
Another problem faced by the view of WMC as resistance to interference is the absence of a relationship between $\mathrm{WMC}$ and the changing-state effect. The results seem to indicate that WMC more specifically measures an ability to control "attention switching" (cf. Berti \& Schröger, 2003; Lavie \& de Fockert, 2005) and, perhaps, retrieval of items from secondary memory (Unsworth \& Engle, 2007), not a domain-general resistance to interference or some other all-purpose pool of attentional resources (cf. Sörqvist, Ljungberg, \& Ljung, 2010).

\section{AUTHOR NOTE}

The research reported in this article was funded by the University of Gävle and a grant from the Swedish Research Council awarded to Staffan Hygge. Parts of the investigation reported here were presented at the Eighth Annual Auditory Perception, Cognition, and Action Meeting in Boston (November 19, 2009). I thank Niklas Halin for assistance in data collection and three anonymous reviewers for many helpful suggestions and comments. Correspondence concerning this article should be addressed to P. Sörqvist, Center for Built Environment, University of Gävle, SE-801 76 Gävle, Sweden (e-mail: patrik .sorqvist@hig.se).

\section{REFERENCES}

BeAman, C. P. (2004). The irrelevant sound phenomenon revisited: What role for working memory capacity? Journal of Experimental Psychology: Learning, Memory, \& Cognition, 30, 1106-1118.

BERTI, S., \& SCHRöGER, E. (2003). Working memory controls involuntary attention switching: Evidence from an auditory distraction paradigm. European Journal of Neuroscience, 17, 1119-1122.

Colle, H. A., \& Welsh, A. (1976). Acoustic masking in primary memory. Journal of Verbal Learning \& Verbal Behavior, 15, 17-31.

Conway, A. R. A., Cowan, N., \& Bunting, M. F. (2001). The cocktail party phenomenon revisited: The importance of working memory capacity. Psychonomic Bulletin \& Review, 8, 331-335.

Conway, A. R. A., Kane, M. J., Bunting, M. F., Hambrick, D. Z., Wilhelm, O., \& Engle, R. W. (2005). Working memory span tasks: A methodological review and user's guide. Psychonomic Bulletin \& Review, 12, 769-786.

Cowan, N. (1995). Attention and memory: An integrated framework. New York: Oxford University Press.

Cowan, N. (2005). Working memory capacity. New York: Psychology Press.

Cronbach, L. J., \& Furby, L. (1970). How we should measure "change": Or should we? Psychological Bulletin, 74, 68-80.

Debener, S., Kranczioch, C., Herrmann, C. S., \& Engel, A. K. (2002). Auditory novelty oddball allows reliable distinction of topdown and bottom-up processes of attention. International Journal of Psychophysiology, 46, 77-84.

EllermeIER, W., \& Zimmer, K. (1997). Individual differences in susceptibility to the "irrelevant speech effect." Journal of the Acoustical Society of America, 102, 2191-2199.

Elliott, E. M., Barrilleaux, K. M., \& Cowan, N. (2006). Individual differences in the ability to avoid distracting sounds. European Journal of Cognitive Psychology, 18, 90-108.

Elliott, E. M., \& Cowan, N. (2005). Coherence of the irrelevant-sound effect: Individual profiles of short-term memory and susceptibility to task-irrelevant materials. Memory \& Cognition, 33, 664-675.

ENGLE, R. W. (2002). Working memory capacity as executive attention. Current Directions in Psychological Science, 11, 19-23.

Friedman, D., Cycowicz, Y. M., \& Gaeta, H. (2001). The novelty P3: An event-related brain potential (ERP) sign of the brain's evaluation of novelty. Neuroscience \& Biobehavioral Reviews, 25, 355-373.

Heitz, R. P., \& EnGLE, R. W. (2007). Focusing the spotlight: Individual differences in visual attention control. Journal of Experimental Psychology: General, 136, 217-240.

Hughes, R. W., VAchon, F., \& Jones, D. M. (2005). Auditory attentional capture during serial recall: Violations at encoding of an algorithm- 
based neural model? Journal of Experimental Psychology: Learning, Memory, \& Cognition, 31, 736-749.

Hughes, R. W., VACHON, F., \& Jones, D. M. (2007). Disruption of shortterm memory by changing and deviant sounds: Support for a duplexmechanism account of auditory distraction. Journal of Experimental Psychology: Learning, Memory, \& Cognition, 33, 1050-1061.

JoNES, D. M., \& MACKEN, W. J. (1993). Irrelevant tones produce an irrelevant speech effect: Implications for phonological coding in working memory. Journal of Experimental Psychology: Learning, Memory, \& Cognition, 19, 369-381.

Jones, D. M., Macken, W. J., \& Mosdell, N. (1997). The role of habituation in the disruption of recall performance by irrelevant sound. British Journal of Psychology, 88, 549-564.

Kane, M. J., Bleckley, M. K., Conway, A. R. A., \& Engle, R. W. (2001). A controlled-attention view of working-memory capacity. Journal of Experimental Psychology: General, 130, 169-183.

KANE, M. J., \& ENGLE, R. W. (2003). Working-memory capacity and the control of attention: The contributions of goal neglect, response competition, and task set to Stroop interference. Journal of Experimental Psychology: General, 132, 47-70.

LANGE, E. B. (2005). Disruption of attention by irrelevant stimuli in serial recall. Journal of Memory \& Language, 53, 513-531.

LAVIE, N., \& DE FocKeRT, J. (2005). The role of working memory in attentional capture. Psychonomic Bulletin \& Review, 12, 669-674

Lustig, C., Hasher, L., \& ZaCKs, R. T. (2007). Inhibitory deficit theory: Recent developments in a "new view." In D. S. Gorfein \& C. M. MacLeod (Eds.), Inhibition in cognition (pp. 145-162). Washington, DC: American Psychological Association.

Macken, W. J., Phelps, F. G., \& Jones, D. M. (2009). What causes auditory distraction? Psychonomic Bulletin \& Review, 16, 139-144.

Macken, W. [J.], Tremblay, S., Alford, D., \& Jones, D. (1999). Attentional selectivity in short-term memory: Similarity of process, not similarity of content, determines disruption. International Journal of Psychology, 34, 322-327.

NeATH, I. (2000). Modeling the effects of irrelevant speech on memory. Psychonomic Bulletin \& Review, 7, 403-423.

Neath, I., Farley, L. A., \& Surprenant, A. M. (2003). Directly assessing the relationship between irrelevant speech and articulatory suppression. Quarterly Journal of Experimental Psychology, 56A, 1269-1278.

Öhman, A. (1979). The orienting response, attention, and learning: An information-processing perspective. In H. D. Kimmel, E. H. van Olst, \& J. F. Orlebeke (Eds.), The orienting reflex in humans (pp. 443-471). Hillsdale, NJ: Erlbaum.

Parmentier, F. B. R. (2008). Towards a cognitive model of distraction by auditory novelty: The role of involuntary attention capture and semantic processing. Cognition, 109, 345-362.
Perham, N., Banbury, S. P., \& Jones, D. M. (2007). Reduction in auditory distraction by retrieval strategy. Memory, 15, 465-473.

REDICK, T. S., \& ENGLE, R. W. (2006). Working memory capacity and attention network test performance. Applied Cognitive Psychology, 20, 713-721.

Salamé, P., \& Baddeley, A. D. (1982). Disruption of short-term memory by unattended speech: Implications for the structure of working memory. Journal of Verbal Learning \& Verbal Behavior, 21, 150164.

SidDLE, D. A. T. (1991). Orienting, habituation, and resource allocation: An associative analysis. Psychophysiology, 28, 245-259.

Sikström, S., \& SöDERLUnd, G. (2007). Stimulus-dependent dopamine release in attention-deficit/hyperactivity disorder. Psychological Review, 114, 1047-1075.

SoKolov, E. N. (1963). Perception and the conditioned reflex. London: Pergamon.

SöRQVIST, P. (2010). Effects of aircraft noise and speech on prose memory: What role for working memory capacity? Journal of Environmental Psychology, 30, 112-118.

Sörqvist, P., Halin, N., \& HygGe, S. (2010). Individual differences in susceptibility to the effects of speech on reading comprehension. Applied Cognitive Psychology, 24, 67-76.

SörQvist, P., LJUngberG, J. K., \& LJUNG, R. (2010). A sub-process view of working memory capacity: Evidence from effects of speech on prose memory. Memory, 18, 310-326.

Tremblay, S., \& Jones, D. M. (1998). Role of habituation in the irrelevant sound effect: Evidence from the effects of token set size and rate of transition. Journal of Experimental Psychology: Learning, Memory, \& Cognition, 24, 659-671.

Tremblay, S., Nicholls, A. P., Alford, D., \& Jones, D. M. (2000). The irrelevant sound effect: Does speech play a special role? Journal of Experimental Psychology: Learning, Memory, \& Cognition, 26, $1750-1754$

Turner, M. L., \& Engle, R. W. (1989). Is working memory capacity task dependent? Journal of Memory \& Language, 28, 127-154.

Unsworth, N., \& ENGLE, R. W. (2007). The nature of individual differences in working memory capacity: Active maintenance in primary memory and controlled search from secondary memory. Psychological Review, 114, 104-132.

Unsworth, N., Schrock, J. C., \& EngLe, R. W. (2004). Working memory capacity and the antisaccade task: Individual differences in voluntary saccade control. Journal of Experimental Psychology: Learning, Memory, \& Cognition, 30, 1302-1321.

(Manuscript received June 1, 2009; revision accepted for publication January 10, 2010.) 